\title{
Double Ion Implantation and Pulsed Laser Melting Processes for Third Generation Solar Cells
}

\author{
Eric García-Hemme, ${ }^{1,2}$ Rodrigo García-Hernansanz, ${ }^{1}$ Javier Olea, ${ }^{2,3}$ \\ David Pastor, ${ }^{1,2,3}$ Álvaro del Prado, ${ }^{1}$ Ignacio Mártil,, ${ }^{1,2}$ Perla Wahnón, ${ }^{2,3,4}$ \\ Kefrén Sanchez, ${ }^{3}$ Pablo Palacios, ${ }^{3,5}$ and Germán González-Díaz ${ }^{1}$ \\ ${ }^{1}$ Departamento Física Aplicada III, Universidad Complutense de Madrid, Ciudad Universitaria, 28040 Madrid, Spain \\ ${ }^{2}$ CEI Campus Moncloa, UCM-UPM, 28040 Madrid, Spain \\ ${ }^{3}$ Instituto de Energía Solar, E.T.S.I. Telecomunicación, Universidad Politécnica de Madrid, Ciudad Universitaria, 28040 Madrid, Spain \\ ${ }^{4}$ Department of TEAT, ETSI Telecomunicación, Universidad Politécnica de Madrid, Ciudad Universitaria, 28040 Madrid, Spain \\ ${ }^{5}$ Department of FyQATA, EIAE, Universidad Politécnica de Madrid, Plaza Cardenal Cisneros 3, 28040 Madrid, Spain
}

Correspondence should be addressed to Eric García-Hemme; eric.garcia@ucm.es

Received 27 February 2013; Accepted 18 October 2013

Academic Editor: Junsin Yi

Copyright (C) 2013 Eric García-Hemme et al. This is an open access article distributed under the Creative Commons Attribution License, which permits unrestricted use, distribution, and reproduction in any medium, provided the original work is properly cited.

\begin{abstract}
In the framework of the third generation of photovoltaic devices, the intermediate band solar cell is one of the possible candidates to reach higher efficiencies with a lower processing cost. In this work, we introduce a novel processing method based on a double ion implantation and, subsequently, a pulsed laser melting (PLM) process to obtain thicker layers of Ti supersaturated Si. We perform ab initio theoretical calculations of Si impurified with $\mathrm{Ti}$ showing that $\mathrm{Ti}$ in $\mathrm{Si}$ is a good candidate to theoretically form an intermediate band material in the Ti supersaturated Si. From time-of-flight secondary ion mass spectroscopy measurements, we confirm that we have obtained a Ti implanted and PLM thicker layer of $135 \mathrm{~nm}$. Transmission electron microscopy reveals a single crystalline structure whilst the electrical characterization confirms the transport properties of an intermediate band material/Si substrate junction. High subbandgap absorption has been measured, obtaining an approximate value of $10^{4} \mathrm{~cm}^{-1}$ in the photons energy range from 1.1 to $0.6 \mathrm{eV}$.
\end{abstract}

\section{Introduction}

Currently our energy model has proven once and again to be incompatible with a sustainable development of our society. Massive fossil fuels consumption has environmentally jeopardized our planet. For this reason, the scientific community is devoting a tremendous effort to the development of new energetic alternatives based on renewable energy sources. Specifically, in the field of photovoltaic solar energy, a third generation of solar cells based on news concepts to increase the efficiency of solar cells is emerging. The intermediate band solar cell (IBSC) is one of these candidates [1]. The concept was first proposed in 1997 [2], and, since then, an important work has been dedicated to describe its physical properties and to produce practical devices that can demonstrate its operational principles $[3,4]$.
An IBSC is based on an intermediate band (IB) material. This new material consists of a semiconductor with its standard conduction and valence band, having a new energy band of allowed states that takes place within the semiconductor bandgap. With this scheme, this kind of material would be able to take advantage of the sub-bandgap photons to promote charge carriers from the valence band to the IB and from the IB to the conduction band. In this sense, the IB would work as an intermediate step, allowing for an increase in the final solar cell photocurrent and, as a consequence, for a higher efficiency.

There exist some different manufacturing approaches to obtain an IB material, like the quantum dots [5] or the highly mismatched alloys [6]. In this paper, we are going to deal with the deep levels impurities approach. It consists in the introduction of a great amount of deep levels 
impurities in a host semiconductor material. Deep levels impurities are well known to create discrete deep energy levels in the bandgap of the host semiconductor. However, a different phenomenon takes place when the concentration of impurities overcomes the so called Mott transition limit [7]. Due to the closer spatial proximity between impurities and to the Pauli Exclusion Principle, an energy splitting of the discrete deep levels would occur. This phenomenon would lead to a localization-delocalization transition similar to the insulator-metal transition and, thus, a band of allowed states would be formed within the band gap of the host semiconductor. As a consequence of this new band of allowed states, the nonradiative recombination would be reduced. This concentration limit has been theoretically calculated to be approximately $6 \times 10^{19} \mathrm{~cm}^{-3}[8]$.

In this research, we have chosen $\mathrm{Si}$ as host semiconductor, since the know-how of the $\mathrm{Si}$ solar cell industry is very mature. As impurity we have chosen $\mathrm{Ti}$, because theoretical studies have shown it to be an excellent candidate to form an IB in Si [9].

At this point, an important technological challenge appears. The concentration limit to form an IB is 5 orders of magnitude over the solid solubility limit of Ti in $\mathrm{Si}\left(10^{14} \mathrm{~cm}^{-3}\right)$ [10]. Fabrication techniques in the thermodynamic equilibrium would not be suitable to overcome this challenge. To get over these difficulties, ion implantation and pulsed laser melting (PLM) processes are the suitable techniques. By means of ion implantation we, are going to be able to introduce in the Si lattice the high Ti concentration required. PLM is the most recommended process in order to recover the crystalline structure after ion implantation, thus allowing for a higher solute trapping of Ti in the Si lattice [11].

Regarding this issue we have achieved important results in the study of Ti implanted and subsequently PLM Si layers. Structurally, we have obtained a higher crystal quality in the final processed material [12]. We have exhaustively characterized electrically the Ti implanted-Si substrate bilayer, finding unusual electrical transport properties that have been well explained in the framework of the IB material theory. We have developed an analytical model to successfully explain and simulate the electrical results [13]. Also, by means of the ATLAS code, we have been able to simulate the transport properties of the bilayer [14]. Finally, by means of photoconductivity measurements, we have measured an increase in the photoresponse of the material for sub-bandgap photon energies, which has been found to be related to an increase in the carrier lifetime as predicted by the IB theory [15].

However, all these positive results have been obtained for a very thin layer of no more than $70 \mathrm{~nm}$. For any photovoltaic applications, it is convenient to obtain a thicker layer of the active optical material so that it may absorb the highest amount of incident light. In this work, we present a double implantation process at different ion energies in order to obtain a thicker layer of the final processed material. Next, we will discuss the theoretical calculations for Ti hypersaturated $\mathrm{Si}$ as well as the experimental work showing the structural, electrical, and optical properties.

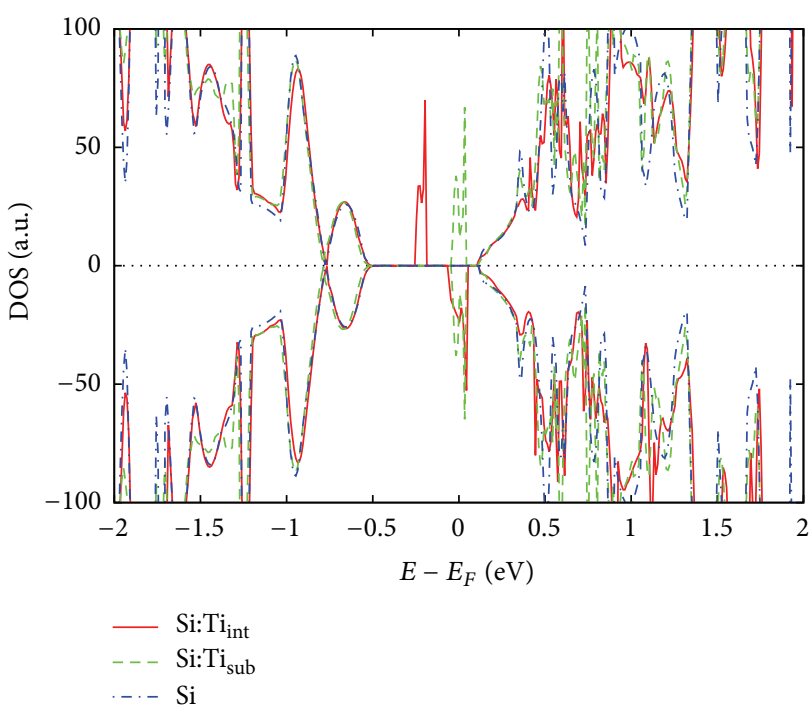

FIGURE 1: Calculated density of states for interstitial $\left(\mathrm{Ti}_{\text {int }}\right)$ and substitutional $\left(\mathrm{Ti}_{\text {sub }}\right) \mathrm{Ti}$ in Si compared with that of bulk Si.

\section{Theoretical Calculations}

An ab-initio theoretical study for Si impurified with $\mathrm{Ti}$ at different concentrations has been made. Here, we present results for a Ti concentration of $10^{20} \mathrm{~cm}^{-3}$ which is the closest to the experimental results. Our calculations were made using spin-polarized Density Functional Theory (DFT) [16, 17] with the generalized gradient (GGA) approximation and the Perdew-Wang 1991 functional [18]. The plane-wave Vienna Ab initio Simulation Package (VASP) code [19] with projector augmented wave (PAW) potentials [20, 21] was used. The valence configurations for the pseudopotentials used in this work were $3 \mathrm{~s} 23 \mathrm{p} 2$ for $\mathrm{Si}$ and $4 \mathrm{~s} 13 \mathrm{~d} 3$ for Ti. Unconstrained relaxations of ions and cells with a convergence tolerance of $0.01 \mathrm{eV} / \mathrm{Ang}$ for atomic forces were carried out for all systems. From the resulting converging wave functions, electronic and optical properties were computed [22]. The energy cutoff established for the basis set was $245 \mathrm{eV}$. The sampling used to describe the Brillouin zone for a 512 atom cell, which corresponds to the abovementioned Ti concentration, was a $\Gamma$ centered $2 \times 2 \times 2$ grid. Detailed energy balances have been also presented [22].

Our results show that although both interstitial and substitutional Ti implantation processes are thermodynamically unfavorable, interstitial $\mathrm{Ti}$ is less unstable. Here, we present in Figure 1 the electronic density of states (DOS) diagram for substitutional and interstitial Ti configurations compared with semiconductor Si spectra. The substitutional Ti DOS diagram shows two empty eg-type doublets which appear approximately at $0.5 \mathrm{eV}$ above the valence band. However, these empty states are not consistent with the experimental results that show an extremely high carrier concentration related to $\mathrm{Ti}$. The interstitially implanted $\mathrm{Ti}$ atoms relax into sites which give a majority spin completely filled triplets and a partially filled minority spins triplets at a higher energy level within the band gap. As a consequence, 
this theoretical result predicts that the presence of interstitial atoms can generate a semifilled band which is compatible with the experimental findings [23] which complies with the characteristic requirements of an appropriate IB photovoltaic material.

\section{Materials and Methods}

Samples $1 \times 1 \mathrm{~cm}^{2}$ in size of $\mathrm{n}$-type $\mathrm{Si}$ (111) with a thickness of $300 \mu \mathrm{m}\left(\rho \approx 200 \Omega \mathrm{cm} ; \mu \approx 1500 \mathrm{~cm}^{2} \mathrm{~V}^{-1} \mathrm{~s}^{-1}\right.$; and $n \approx$ $2 \times 10^{13} \mathrm{~cm}^{-3}$ at RT) were implanted in a refurbished VARIAN CF3000 Ion Implanter. One set was single Ti implanted at $25 \mathrm{keV}$ with a $10^{15} \mathrm{~cm}^{-2}$ dose whilst the other set was double Ti implanted at $35 \mathrm{keV}$ and $150 \mathrm{keV}$ with doses of $10^{15} \mathrm{~cm}^{-2}$ and $4 \times 10^{15} \mathrm{~cm}^{-2}$, respectively. All samples were implanted using a $7^{\circ}$ tilt angle to reduce possible channeling effects. After implantation, all the samples were PLM processed with one $20 \mathrm{~ns}$ long pulse at an energy density of $0.8 \mathrm{~J} / \mathrm{cm}^{2}$ for the single implantation case and $1.8 \mathrm{~J} / \mathrm{cm}^{2}$ for the double implanted samples. The system was a $\mathrm{KrF}$ excimer laser $(248 \mathrm{~nm})$ at IPG Photonics (New Hampshire, USA). Finally, we e-beam evaporated 4 contacts in the corners sample to electrically measure it using the van der Pauw method. The contacts were made of $100 \mathrm{~nm}$ Ti plus $200 \mathrm{~nm} \mathrm{Al}$.

Electrical characterization was made at variable temperature $(100-300 \mathrm{~K})$ placing the samples inside a homemade liquid nitrogen cryostat. In order to avoid moisture condensation, the cryostat was attached to a vacuum pump. A Keithley SCS 4200 model with four source and measure units was used to perform the sheet resistance and Hall effect measurement with the van der Pauw configuration. This assumes a parallel electrical conduction scheme. Namely, the current flows between electrodes both through the processed layer and the Si substrate. To analyze the Ti depth profile, time-of-flight secondary ion mass spectrometry (ToF-SIMS) measurements were carried out in a ToF-SIMS IV model manufactured by ION-TOF, with a $25 \mathrm{keV}$ pulsed $\mathrm{Bi}^{3+}$ beam at $45^{\circ}$ incidence. A $10 \mathrm{keV}$ voltage was used to extract the secondary ions generated, and their time of flight from the sample to the detector was measured with a reflection mass spectrometer. Cross sectional transmission electron microscopy (TEM) images were obtained by a JEOL-2010 working at $200 \mathrm{keV}$ and by a JEOL JEM-2000FX working at $200 \mathrm{keV}$ at C.A.I. de Microscopía (Madrid, Spain). Simultaneously with the TEM measurements, electron diffraction (ED) patterns with a selected area of diffraction of about $50 \mathrm{~nm}$ were obtained. The ED images provided information of the crystalline morphology of the implanted layer. Optical characterization was carried out by transmittance and reflectance measurements using a Perkin Elmer Lambda 9 UV-VIS-IR spectrophotometer in the $0.55-1.2 \mathrm{eV}$ range. Additionally, for reflectance measurements, we used an integrating sphere photodetector in the same energy range.

\section{Results and Discussion}

In Figure 2, we can observe the $\mathrm{Ti}$ depth profile obtained from ToF-SIMS measurements of the two sets of implanted

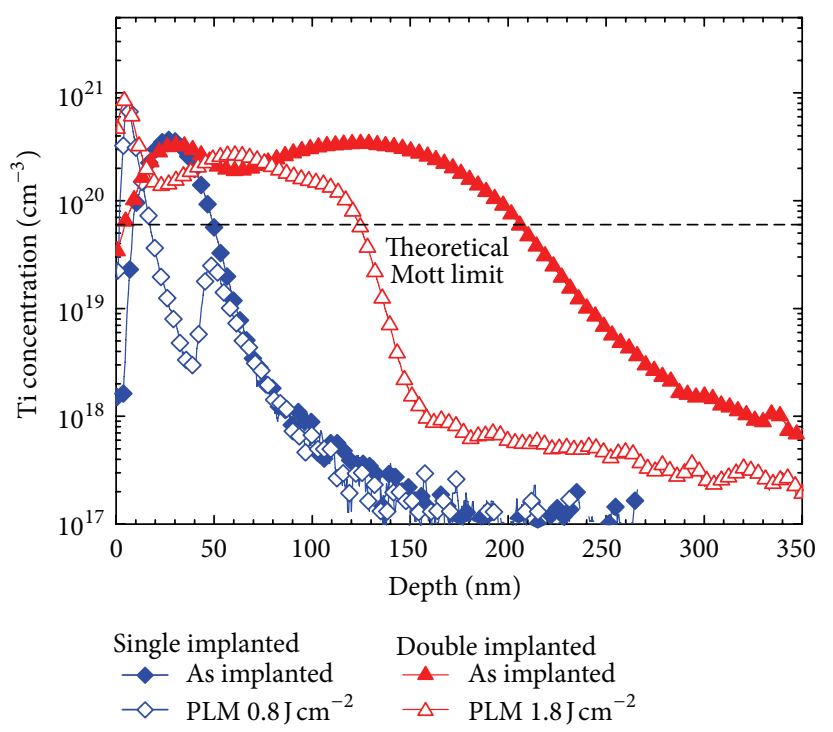

Figure 2: ToF-SIMS Ti concentration depth profile for the single implanted samples and for the double implanted samples before and after PLM. The single implanted set of samples were PLM processed at $0.8 \mathrm{Jcm}^{-2}$ and the double implanted were PLM processed at $1.8 \mathrm{Jcm}^{-2}$.

samples before and after the PLM process. We can observe that for the two full processed samples we obtain a $\mathrm{Ti}$ concentration over the Mott limit and then, theoretically, an IB material would be formed in the top layer. Specifically, we obtain a Ti concentration around $4 \times 10^{20} \mathrm{~cm}^{-3}$ in a layer of $20 \mathrm{~nm}$ for the single implanted sample and a thicker layer of $135 \mathrm{~nm}$ for the double implanted sample. In this figure, we can appreciate the effect of the PLM process since we can observe a push out effect of the Ti profile towards the surface of the $\mathrm{Si}$ substrate. In spite of this push out effect, the Ti concentration that remains in the $\mathrm{Si}$ lattice is well above the theoretical limit to form an IB material.

Since, the solid solubility limit of $\mathrm{Ti}$ on $\mathrm{Si}$ has been surpassed in 5 orders of magnitude, it would be interesting to study the crystalline quality of the processed samples. Figures 3(a) and 3(b) show TEM images for the single implanted and double implanted sample, respectively. We can observe in Figure 3(a) an excellent crystalline structure for the $10^{15} \mathrm{~cm}^{-2}$ single implanted samples after the PLM process. A single crystalline layer is obtained in spite of the high Ti concentration ( 5 orders of magnitude over the solid solubility limit). In addition, no differences between the electron diffraction pattern of the implanted layer and the Si substrate are observed. However, this crystalline Ti supersaturated $\mathrm{Si}$ layer with a Ti concentration above the Mott limit is obtained in a thin layer of $20 \mathrm{~nm}$. From the ED pattern in Figure 3(b), we observe that the double implanted layer presents also a single crystalline structure; however, from TEM images, we observe that some regions appear slightly defective. We observe that this slightly defective layer presents a thickness of $123 \mathrm{~nm}$. This thickness corresponds with the observed depth in Figure 2 that presents a Ti concentration over the Mott limit. Additionally, in a detailed Raman spectroscopy 


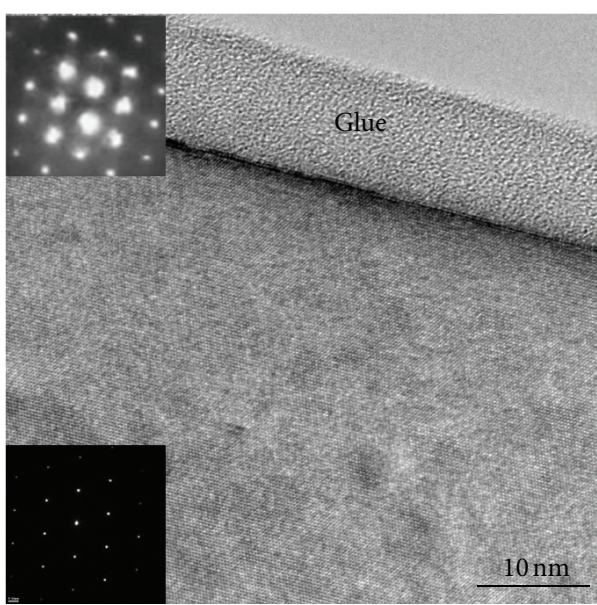

(a)

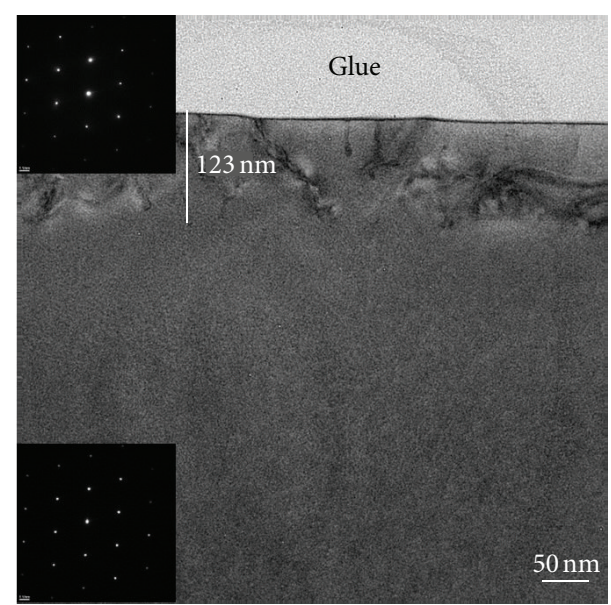

(b)

FIGURE 3: TEM and ED pattern of the two implanted and PLM processed samples; (a) corresponds with the single implanted while (b) corresponds with the double implanted samples.

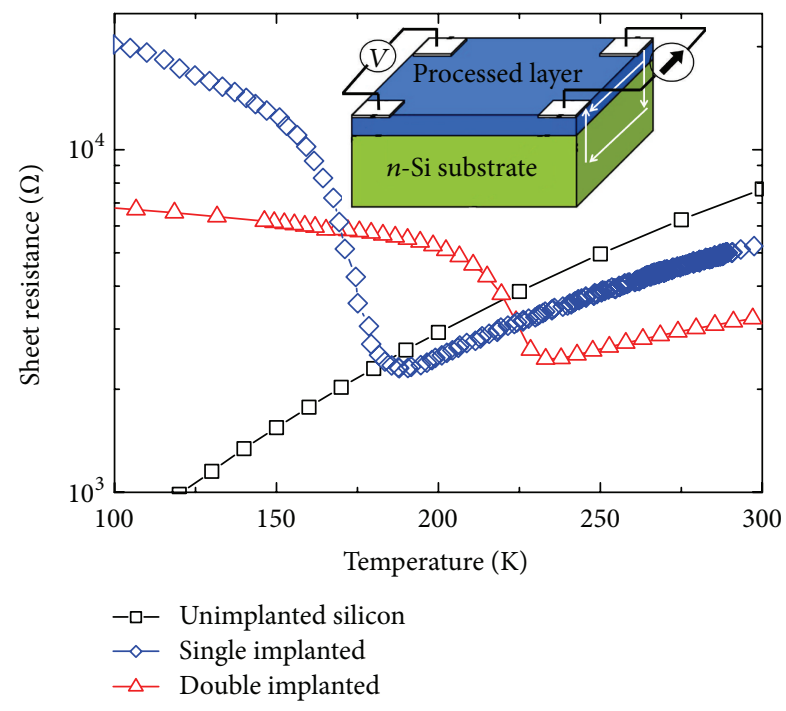

FIGURE 4: Sheet resistance as a function of temperature measured for the single Ti implanted and the double Ti implanted samples. A Si reference substrate is used for comparison purposes. Inset shows a schematic 3D view of the final processed sample and the van der Pauw set-up for electrical characterization.

characterization, no evidence of secondary phase formation (like Ti silicides) has been observed in samples with similar Ti concentrations [24].

As commented above, the electronic transport properties can give us information about the IB formation. In Figure 4, we represent the sheet resistance of the two implanted samples and of a Si reference substrate as a function of the temperature. In the inset of Figure 4, we present a schematic $3 \mathrm{D}$ view of the van der Pauw set-up used for the sheet resistance measurements. For the Si reference sample, we observe the expected behavior of the decrease of the sheet resistance as the temperature decreases due to the reduction of the optical phonon scattering. However, the two sets of implanted samples show an unusual behavior. At a given temperature of $225 \mathrm{~K}$ for the double implanted sample and of $180 \mathrm{~K}$ for the single implanted one, the sheet resistance begins to increase over the sheet resistance of the Si substrate as the temperature decreases. This is not the expected behavior if we assume a parallel electrical conduction scheme (implanted layer-Si substrate) since the sheet resistance of the bilayer cannot exceed the sheet resistance of one of the conduction layer (Si reference substrate). Then, an electrical decoupling between the implanted layer and the substrate seems to take place as we are only contacting electrically the Ti implanted layer. This electrical decoupling effect has been related to the properties of the bilayer formed by the IB material and the Si substrate [25]. A transversal electrical resistance between the two layers increases as the temperature decreases and, at a low enough temperature, the electrical transport between the two layers is completely blocked. Then, at low temperatures, we are only measuring the transport properties of the IB material.

In Figure 5, we can observe the Hall mobility as a function of the temperature for the two implanted samples and for a $\mathrm{Si}$ reference substrate. The sign of the Hall voltage is negative, resulting in electron mobility for all the temperatures. We observe the expected tendency of an increase in the mobility as the temperature decreases. However, as it was the case with the sheet resistance, we observe that the two implanted samples show a different behavior at the same temperature of $225 \mathrm{~K}$ for the double implanted sample and of $180 \mathrm{~K}$ for the single implanted one. An important decrease in mobility appears as the temperature decreases, reaching a small, an almost constant, value. This performance has been also explained as an electrical decoupling of the bilayer in correspondence with the electrical decoupling quoted above for the sheet resistance. Thus, the tendency of the mobility measurements indicates that only the mobility of the Ti implanted layer for temperatures low enough is measured [26]. 


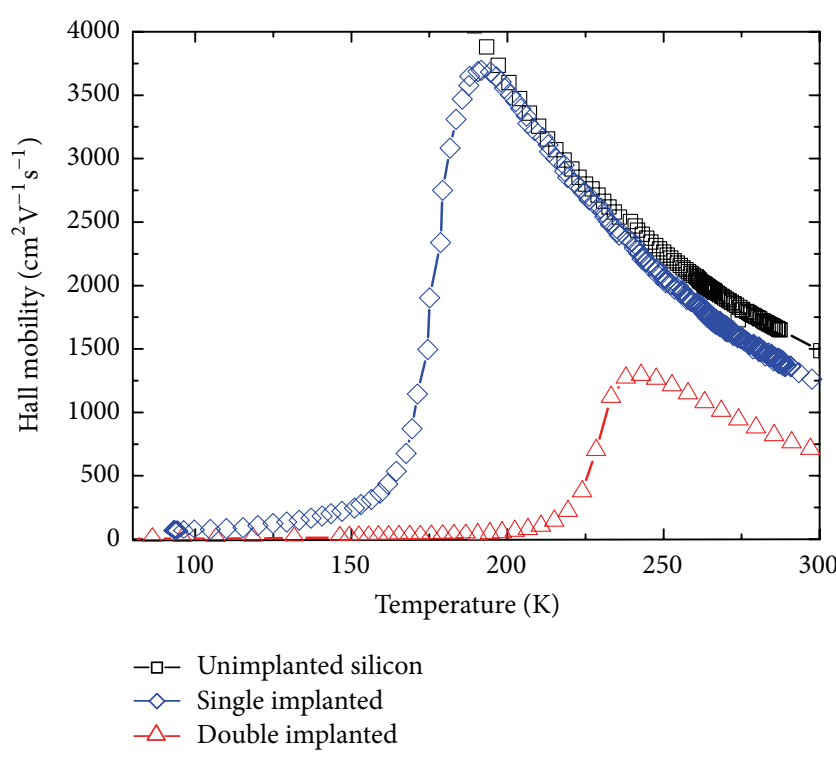

FIGURE 5: Hall mobility as a function of temperature measured for the single implanted and the double implanted samples. A Si reference substrate is used for comparison purposes.

The authors have previously developed an analytical model by means of which this electrical decoupling process has been studied. This analytical model is implemented according with the theoretical electrical transport properties of an IB material-Si substrate bilayer [13]. Since we have been able to associate the electrical decoupling process with the formation of an IB material, we can suggest that the IB material has been obtained for both technological processes, but for different thicknesses. The technical details of the analytical model and the simulation can be obtained from $[13,25,26]$.

Although the electrical characterization points to the IB formation in the implanted layer by means of the analytical model, an optical characterization is required to elucidate if this material could have a potential photovoltaic use. In Figure 6, we show the product $\alpha_{\mathrm{il}} w_{\mathrm{il}}$ for the two samples, according to the mathematical procedure showed in [27]. $\alpha_{\mathrm{il}}$ refers to the absorption coefficient on the implanted layer and $w_{\mathrm{il}}$ refers to the thickness of the implanted layer. We observe that the two samples exhibit notable sub-bandgap absorption. This sub-bandgap absorption cannot be related with defects, since by TEM measurements we have observed an almost perfect lattice reconstruction after the PLM process (Figures 3(a)-3(b)). Moreover, in [27], we have observed that more defective samples present less absorption than samples with good crystalline quality, thus suggesting that this high sub-bandgap absorption is not related with the presence of defects, nor can this absorption be explained by free carrier absorption, since this mechanism has dependence with the sub-bandgap photon energy opposite to the one shown in Figure 6 [28-30]. Then, this optical absorption could be related with the formation of an IB in the processed layer, which might be increasing the absorption process through optical transitions involving the IB. It is important to note that

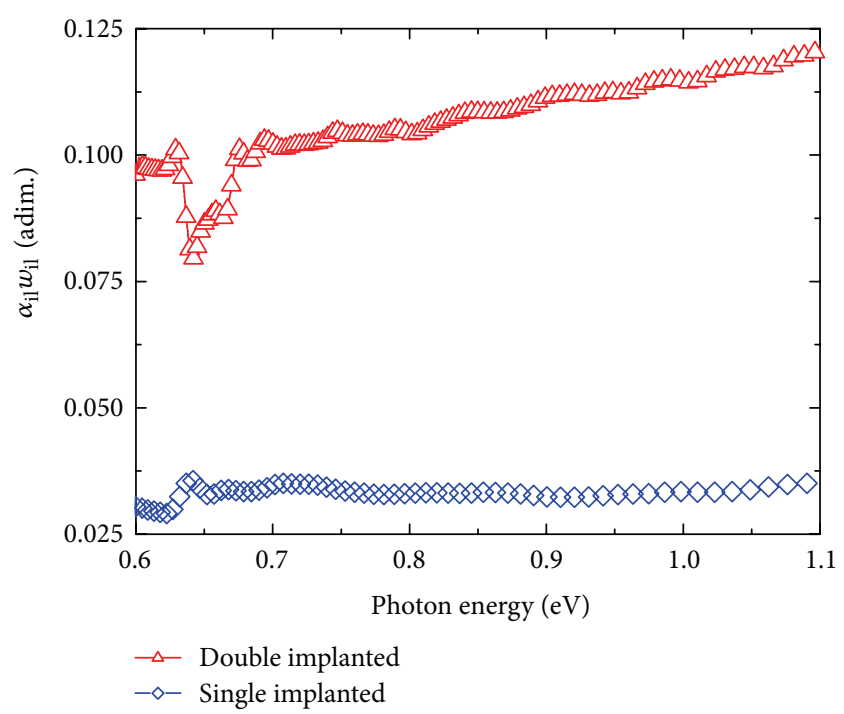

FIgURE 6: Product of the absorption coefficient of the implanted layer $\left(\alpha_{\mathrm{il}}\right)$ and the thickness of the implanted layer $\left(w_{\mathrm{il}}\right)$ as a function of subbandgap photon energies for the single implanted and the double implanted samples.

the absorption coefficient for a pure Si sample is negligibly in the sub-bandgap region. Finally, we can estimate a value for the absorption coefficient using the thickness of implanted layer that presents $\mathrm{Ti}$ concentrations over the theoretical limit to form an IB material. From Figure 2, we can estimate $20 \mathrm{~nm}$ for the single implanted and $135 \mathrm{~nm}$ for the double implanted sample. From this approximation, we can deduce an absorption coefficient of about $10^{4} \mathrm{~cm}^{-1}$ for both samples on Figure 6. This high sub-bandgap absorption would be an important positive feature to take advantage from in any future photovoltaic application.

\section{Conclusions}

In conclusion, a double ion implantation followed by a PLM process has been presented in order to obtain thicker layers of IB material. Ab-initio theoretical calculations predict the formation of an IB material in Ti supersaturated Si. The structural, electrical, and optical properties of the double implanted layers have been compared with those of the thinner layers obtained by a single implantation process. By ToF-SIMS, these layers show a $\mathrm{Ti}$ concentration well above the theoretical limit to form an IB material. TEM and ED patterns have shown a single crystalline structure, and few structural defects have been observed for the double implanted sample as well as a nearly perfect recovery for the single implanted sample in spite of the high Ti concentration. Sheet resistance and mobility measurements as a function of temperature have shown similar electrical decoupling processes for both sets of samples (single implanted and double implanted samples). By means of the analytical model presented in [13], we have been able to associate the electrical decoupling process to the formation of an IB in the processed layer. Important sub-bandgap absorption has been measured 
for the two sets of samples. All these results point out that we have implemented a double implantation process in order to obtain thicker layers of the IB material with very high crystalline, electrical, and optical qualities. Focusing on a prospective third generation of solar cells, the need for these thicker layers of the active IB material should become mandatory.

\section{Acknowledgments}

The authors would like to acknowledge the C.A.I. de Técnicas Físicas of the Universidad Complutense de Madrid for the ion implantations and metallic evaporations, the Nanotechnology and Surface Analysis Services of the Universidad de Vigo C.A.C.T.I. for ToF-SIMS measurements, and the C.A.I. de Microscopía of the Universidad Complutense de Madrid for TEM measurements. The authors thankfully acknowledge the computer resources, technical expertise, and assistance provided by the Centro de Supercomputación y Visualización de Madrid (CeSViMa) and the Spanish Supercomputing Network. They would like also to thanks Dr. J. Herrero (CIEMAT) for UV-VIS-IR measurements facilities. This work was partially supported by the Project NUMANCIA II (Grant no. S-2009/ENE/1477) funded by the Comunidad de Madrid. Research by Eric García-Hemme was also supported by a PICATA predoctoral fellowship of the Moncloa Campus of International Excellence (UCM-UPM). Javier Olea and David Pastor thank Professor A. Martí and Professor A. Luque for the useful discussions and guidance and acknowledge the financial support from the MICINN within the program Juan de la Cierva (JCI-2011-10402 and JCI-2011-11471), under which this research was undertaken.

\section{References}

[1] A. Luque and A. Martí, "Photovoltaics: towards the intermediate band," Nature Photonics, vol. 5, no. 3, pp. 137-138, 2011.

[2] A. Luque and A. Martí, "Increasing the efficiency of ideal solar cells by photon induced transitions at intermediate levels," Physical Review Letters, vol. 78, no. 26, pp. 5014-5017, 1997.

[3] A. Luque, A. Martí, and C. Stanley, "Understanding intermediate-band solar cells," Nature Photonics, vol. 6, no. 3, pp. 146152, 2012.

[4] N. Ahsan, N. Miyashita, M. Monirul Islam, K. Man Yu, W. Walukiewicz, and Y. Okada, "Two-photon excitation in an intermediate band solar cell structure," Applied Physics Letters, vol. 100, no. 17, Article ID 172111, 2012.

[5] A. Martí, N. López, E. Antolín et al., "Novel semiconductor solar cell structures: the quantum dot intermediate band solar cell," Thin Solid Films, vol. 511-512, pp. 638-644, 2006.

[6] K. M. Yu, M. A. Scarpulla, R. Farshchi, O. D. Dubon, and W. Walukiewicz, "Synthesis of highly mismatched alloys using ion implantation and pulsed laser melting," Nuclear Instruments and Methods in Physics Research, Section B, vol. 261, no. 1-2, pp. 1150-1154, 2007.

[7] N. F. Mott, "The metal-insulator transition in extrinsic semiconductors," Advances in Physics, vol. 21, no. 94, pp. 785-823, 1972.

[8] A. Luque, A. Martí, E. Antolín, and C. Tablero, "Intermediate bands versus levels in non-radiative recombination," Physica $B$, vol. 382, no. 1-2, pp. 320-327, 2006.
[9] K. Sanchez, I. Aguilera, P. Palacios, and P. Wahnon, "Formation of a reliable intermediate band in Si heavily coimplanted with chalcogens (S, Se, Te) and group III elements (B, Al)," Physical Review B, vol. 82, Article ID 165201, 8 pages, 2010.

[10] S. Hocine and D. Mathiot, "Titanium diffusion in silicon," Applied Physics Letters, vol. 53, no. 14, pp. 1269-1271, 1988.

[11] C. W. White, S. R. Wilson, B. R. Appleton, and F. W. Young Jr., "Supersaturated substitutional alloys formed by ion implantation and pulsed laser annealing of group-III and group-V dopants in silicon," Journal of Applied Physics, vol. 51, no. 1, pp. 738-749, 1980.

[12] J. Olea, M. Toledano-Luque, D. Pastor, E. San-Andrés, I. Mártil, and G. González-Díaz, "High quality Ti-implanted Si layers above the Mott limit," Journal of Applied Physics, vol. 107, no. 10, Article ID 103524, 2010.

[13] J. Olea, G. González-Díaz, D. Pastor et al., “Two-layer Hall effect model for intermediate band Ti-implanted silicon," Journal of Applied Physics, vol. 109, Article ID 063718, 8 pages, 2011.

[14] J. Olea, G. González-Díaz, D. Pastor, and I. Mártil, "Electronic transport properties of Ti-impurity band in Si," Journal of Physics D, vol. 42, no. 8, Article ID 085110, 2009.

[15] E. Garcia-Hemme, R. Garcia-Hernansanz, J. Olea et al., "Subbandgap spectral photo-response analysis of Ti supersaturated Si," Applied Physics Letters, vol. 101, Article ID 192101, 2012.

[16] P. Hohenberg and W. Kohn, "Inhomogeneous electron gas," Physical Review, vol. 136, no. 3B, pp. B864-B871, 1964.

[17] W. Kohn and L. J. Sham, "Self-consistent equations including exchange and correlation effects," Physical Review, vol. 140, no. 4A, pp. A1133-A1138, 1965.

[18] J. P. Perdew, J. A. Chevary, S. H. Vosko et al., "Atoms, molecules, solids, and surfaces: applications of the generalized gradient approximation for exchange and correlation," Physical Review $B$, vol. 46, no. 11, pp. 6671-6687, 1992.

[19] G. Kresse and J. Hafner, "Ab initio molecular dynamics for liquid metals," Physical Review B, vol. 47, no. 1, pp. 558-561, 1993.

[20] G. Kresse and D. Joubert, "From ultrasoft pseudopotentials to the projector augmented-wave method," Physical Review B, vol. 59, no. 3, pp. 1758-1775, 1999.

[21] P. E. Blöchl, "Projector augmented-wave method," Physical Review B, vol. 50, no. 24, pp. 17953-17979, 1994.

[22] K. Sanchez, I. Aguilera, P. Palacios, and P. Wahnon, "Assessment through first-principles calculations of an intermediate-band photovoltaic material based on Ti-implanted silicon: Interstitial versus substitutional origin," Physical Review B, vol. 79, Article ID 165203, 7 pages, 2009.

[23] D. Pastor, J. Olea, A. Munoz-Martin, A. Climent-Font, I. Mártil, and G. González-Díaz, "Interstitial Ti for intermediate band formation in Ti-supersaturated silicon," Journal of Applied Physics, vol. 112, no. 11, 2012.

[24] D. Pastor, J. Olea, A. del Prado et al., "UV and visible Raman scattering of ultraheavily Ti implanted Si layers for intermediate band formation," Semiconductor Science and Technology, vol. 26, no. 11, Article ID 115003, 2011.

[25] D. Pastor, J. Olea, A. del Prado et al., "Electrical decoupling effect on intermediate band Ti-implanted silicon layers," Journal of Physics D, vol. 46, Article ID 135108, 2013.

[26] J. Olea, D. Pastor, E. Garcia-Hemme et al., "Low temperature intermediate band metallic behavior in Ti implanted Si," Thin Solid Films, vol. 520, no. 21, pp. 6614-6618, 2012.

[27] J. Olea, Á. del Prado, D. Pastor, I. Mártil, and G. GonzálezDíaz, "Sub-bandgap absorption in Ti implanted Si over the Mott limit," Journal of Applied Physics, vol. 109, Article ID 113541, 2011. 
[28] W. Spitzer and H. Y. Fan, "Infrared absorption in $n$-type silicon," Physical Review, vol. 108, no. 2, pp. 268-271, 1957.

[29] H. Hara and Y. Nishi, "Free carrier absorption in $p$-type silicon," Journal of the Physical Society of Japan, vol. 21, no. 6, p. 1222, 1966.

[30] D. K. Schroder, R. N. Thomas, and J. C. Swartz, "Free carrier absorption in silicon," IEEE Transactions on Electron Devices, vol. 25, pp. 254-261, 1978. 

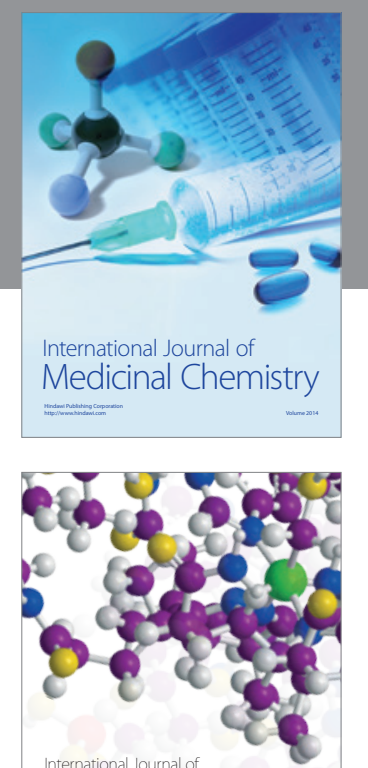

\section{Carbohydrate} Chemistry

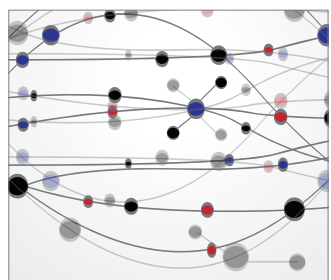

The Scientific World Journal
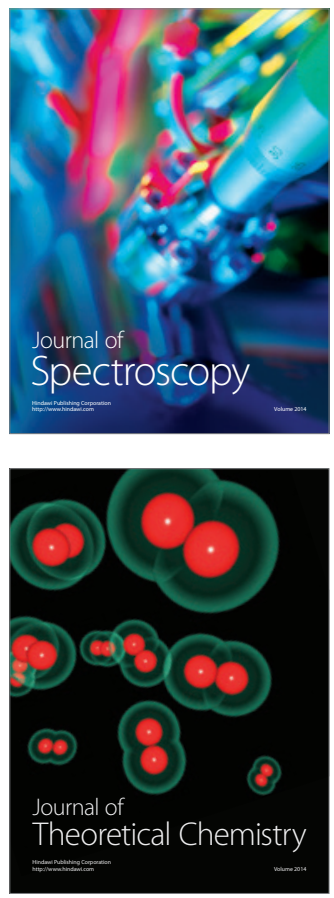
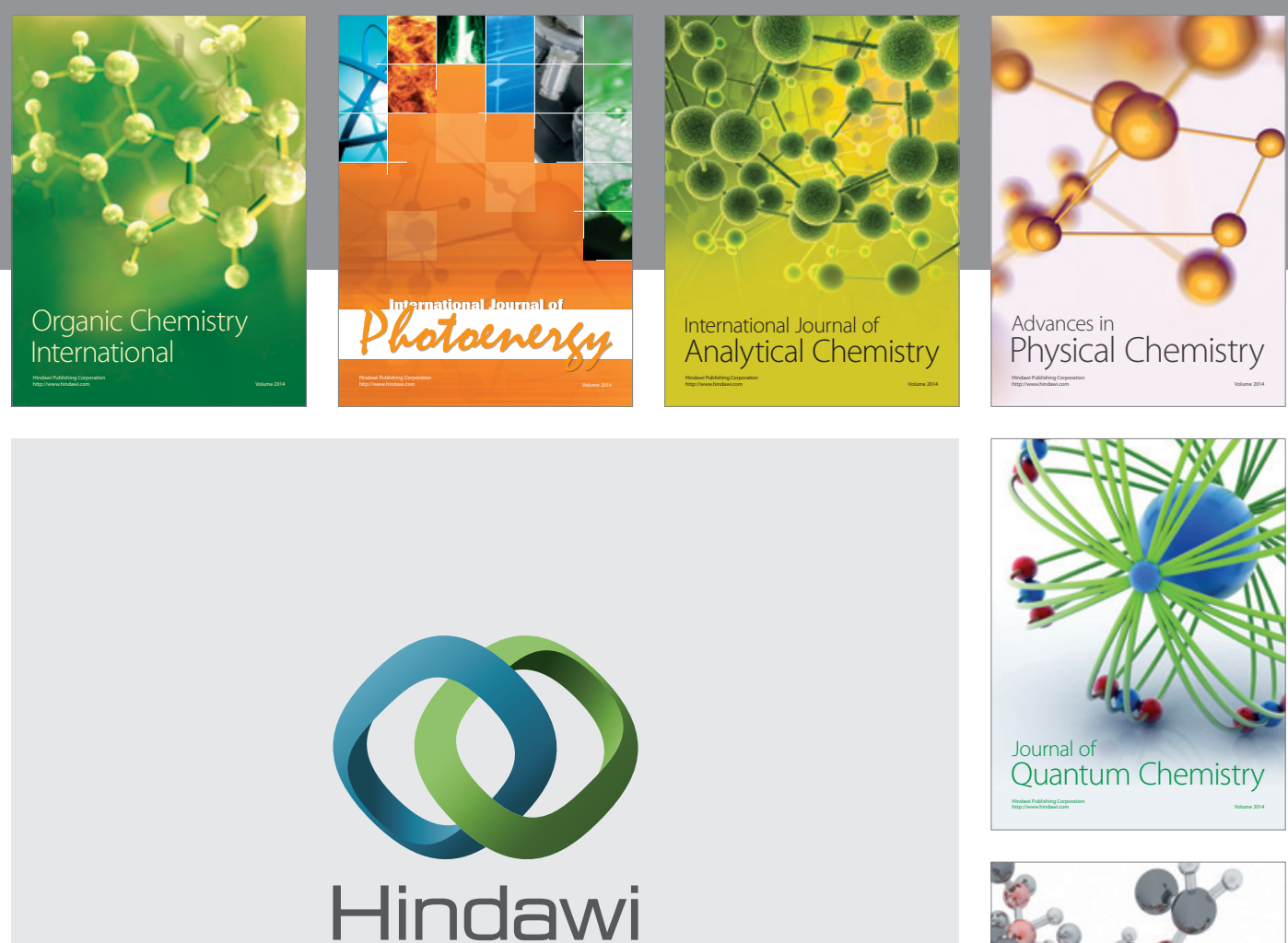

Submit your manuscripts at

http://www.hindawi.com

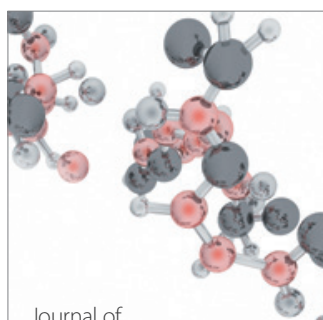

Analytical Methods

in Chemistry

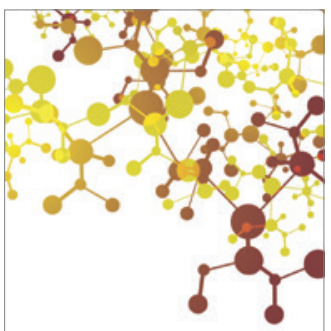

Journal of

Applied Chemistry

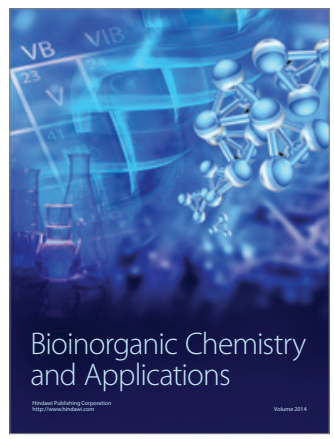

Inorganic Chemistry
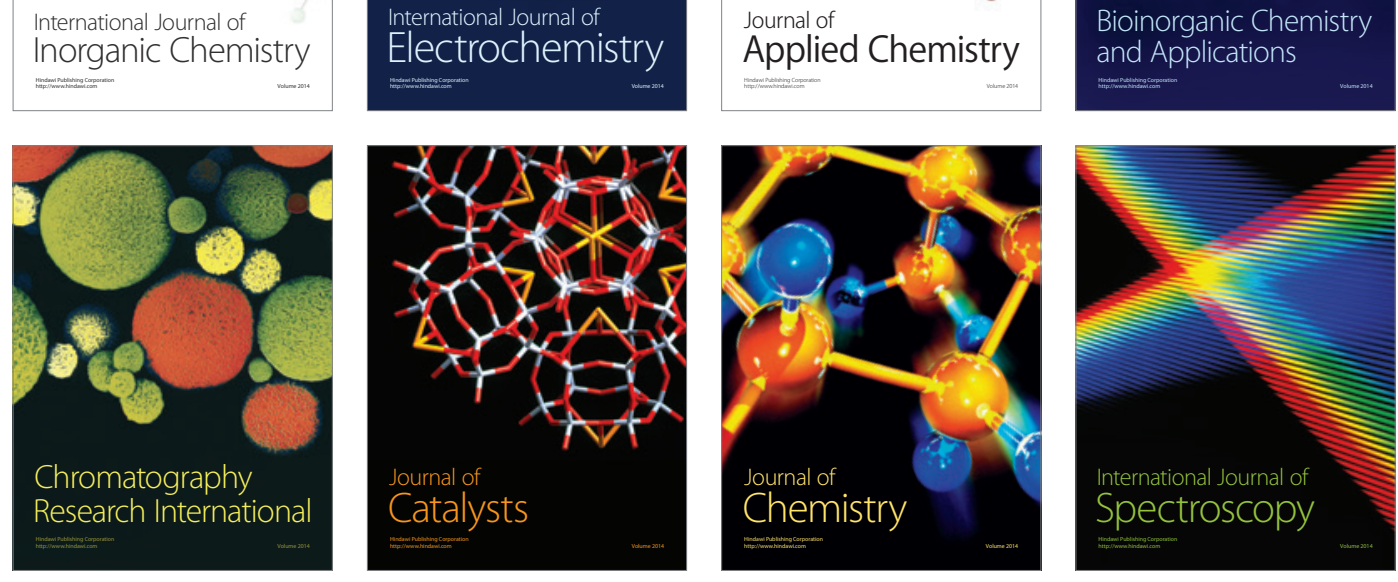